\title{
Special Issue on Charity Accounting, Reporting and Regulation
}

\author{
Carolyn J. Cordery • Gareth G. Morgan
}

Published online: 30 April 2013

(C) International Society for Third-Sector Research and The Johns Hopkins University 2013

In many countries, certain types of third-sector organisations (TSOs) are given formal recognition as charities-which may entitle them to specific kinds of legal protection and/or specific tax concessions. Charities represent a significant subsector of TSOs where the formal recognition is often legislative (for example, the Statute of Charitable Uses (1601) in England formed the cornerstone of the legal definition of charitable purpose in that country). Further the tax concessions allowed to charities and often to their donors have meant that charities and donors have a real interest in the way in which a country legally defines charitable purpose.

Increasingly, discussion in the literature on the third-sector has focussed on the rise of regulation and its effect on organisations. The impetus for regulation and oversight of TSOs arises partly from calls for increased accountability and transparency of the sector. One role of regulation is to enhance the reputation of TSOs through requiring them to meet particular standards in terms of being legally registered and report on a regular basis. The extent of charities' tax concessions, as well as the economic and social potential of philanthropy also provide reasons why governments may seek to use regulatory schemes to monitor these particular organisations. Nevertheless, the design of regulation and a jurisdiction's institutional arrangements will impact the success of such regulation in achieving these aims. Regulation may result in sectoral or independent oversight, but commonly involves government intervention, if only to establish the legal forms which TSOs may take.

\section{J. Cordery}

School of Accounting and Commercial Law, Victoria University, PO Box 600, Wellington 6140,

New Zealand

e-mail: Carolyn.Cordery@vuw.ac.nz

G. G. Morgan ( $₫)$

Sheffield Business School, Sheffield Hallam University, Stoddart Building, City Campus,

Sheffield S1 1WB, UK

e-mail: gareth.morgan@shu.ac.uk 
While the number of charities that are regulated can be large (for instance, around 180,000 registered charities are subject to direct regulatory oversight in England and Wales), these numbers pale into insignificance when compared to all TSOs in the same country. Accordingly, charity regulatory effort is concentrated rather than dispersed, although there is great variety in legally defined charities (in the sectors in which they operate and in their beneficiaries).

Furthermore, while regulators often state their prime raison d'être is to enhance accountability and transparency, this is frequently accomplished through requiring charities to report-to account, rather than specifically to discharge accountability. The requirements in different jurisdictions for charity accounting and reporting are thus a key consideration. In this special issue of Voluntas, a number of different questions are encountered in the discussion of charity regulation and reporting and the political aspects of regulation.

The interpretations of legal requirements by charity regulators and the extent to which they modify their behaviour in relation to feedback from their constituents is the subject of Sinclair and Bolt's study into accounting standard setting for the charitable sector in New Zealand. They ask: 'how can these standard setters effectively develop good reporting?' The New Zealand regulator's use of 'working groups' of salient stakeholders to discern constituent's views was a tactic that superseded vague or incomplete submissions from constituents.

Specific reporting regulation is also the topic of two further articles. Connolly, Hyndman and McConville consider the nexus between a focus on fundraising ratios, accounting standards and charities' reporting. In their study of the financial accounts of large charities in the United Kingdom, the manner in which support and direct charitable costs are allocated is considered before and after an accounting standard change. The practice of many charities in their sample has been to limit disclosure (in contravention of the Statement of Recommended Practice that governs their reporting). It is disappointing that independent parties (the regulator, auditors and large donors) do not appear to have been able to compel charities to comply with these regulations.

Morgan and Fletcher consider reporting in England and Wales. At the heart of being a charity is the requirement to work in the public benefit, so the introduction in that jurisdiction of a requirement for a charity to report on how it has discharged the public benefit requirement appears to be logical. Their study considered practices of small, medium and large registered charities, and the way in which this reporting was perceived to increase accountability discharge. While their study found poor compliance with this requirement, particularly by smaller charities, it also found that requiring charities to reflect and report on how they meet the public benefit requirement was widely seen as a positive aspect of this regulation.

The role of the regulator to improve and monitor reporting is also assumed in Cordery's research into the performance of the New Zealand Charities Commission. There, public trust and confidence in charities has continued to decline and the regulator's light-handed approach has resulted in the publicly available reporting being of poor quality which increases information asymmetry. Cordery argues that there is a need for deterrence methods to increase the likelihood that charities will 
discharge accountability in a transparent manner. Then perhaps, public trust and confidence in charities will increase.

Breen takes a cross-country view of regulatory practices in respect of charity reporting as she supports the argument that good practices will enhance the 'marketplace' for charitable donations. Reviewing the very different practices of charity regulators in the United Kingdom and the United States, and drawing also on practices in Australia and New Zealand, Breen hopes to find principles to guide the emergent Irish regulator. She reflects particularly on the sometimes ambiguous place of International Financial Reporting Standards (IFRS) in charity reporting. Like Cordery she finds that the expectation that charity comparability would be an outcome of widely available reporting is not met, due to the poor quality of that reporting. Strained regulatory capacity impacts the ability of regulators to employ deterrence methods to improve reporting, but this is necessary if effective charity oversight is to occur.

The use of institutionally polycentric mechanisms is a solution suggested by Phillips, who describes the emergent co-regulation in Canada's charitable sector. This innovation aims to improve accountability through more targeted state regulation, charity self-regulation (through certification to a Standards Program) and the rise of an independent third party using Open Data to comment on charity accountability and efficiency. However, Phillips also highlights the risk of regulatory spaces for charity sectors becoming more crowded: she questions whether the increased transparency is focusing on the necessary and appropriate areas of charity accountability and encourages continuing scrutiny as this coregulation matures.

This special issue arose from two panel sessions at the 10th ISTR Conference in Siena and the papers provide an extensive overview of charity regulation in a number of different countries and the role of reporting in increasing accountability or otherwise. ${ }^{1}$ A number of authors have raised the issue of regulatory cost, both on the charities as well as the regulators. In light of reducing budget allowances for regulators to detect and impose sanctions on charities that report poorly, we wonder if these costs will reduce the historically strong impetus for charity regulation and disclosure. Furthermore, in light of the need for regulators and charities to agree on the interpretation of 'public benefit' and allowed activities in the public interest, we suspect there are many debates yet to be had as legal and societal interpretations continue to re-define these terms. We hope regulators will place greater emphasis on requiring charities to report outputs and outcomes in relation to their charitable objects and that this will result in increased communications with beneficiaries as well as donors. Improvements will require the involvement of not only charities and government accounting and charity regulators but also media, charity watchdogs and information facilitators, funders and donors.

\footnotetext{
1 We are grateful for the support of the Editor, Bernard Enjolras, who has handled the reviewing and selection of the final papers for this issue.
} 